\title{
New Vacuum Solar Telescope observations of a flux rope tracked by a filament activation
}

\author{
Shuhong Yang ${ }^{1}$, Jun Zhang ${ }^{1}$, Zhong Liu ${ }^{2}$, and Yongyuan Xiang ${ }^{2}$
}

\begin{abstract}
One main goal of the New Vacuum Solar Telescope (NVST) which is located at the Fuxian Solar Observatory is to image the Sun at high resolution. Based on the high spatial and temporal resolution NVST $\mathrm{H} \alpha$ data and combined with the simultaneous observations from the Solar Dynamics Observatory for the first time, we investigate a flux rope tracked by a filament activation. The filament material is initially located at one end of the flux rope and fills in a section of the rope, and then the filament is activated due to magnetic field cancellation. The activated filament rises and flows along helical threads, tracking out the twisted flux rope structure. The length of the flux rope is about $75 \mathrm{Mm}$, the average width of its individual threads is $1.11 \mathrm{Mm}$, and the estimated twist is $1 \pi$. The flux rope appears as a dark structure in $\mathrm{H} \alpha$ images, a partial dark and partial bright structure in $304 \AA$, while as bright structures in $171 \AA$ and $131 \AA$ images. During this process, the overlying coronal loops are quite steady since the filament is confined within the flux rope and does not erupt successfully. It seems that, for the event in this study, the filament is located and confined within the flux rope threads, instead of being suspended in the dips of twisted magnetic flux.
\end{abstract}

Subject headings: Sun: atmosphere - Sun: evolution — Sun: filaments, prominences - Sun: magnetic fields

\section{INTRODUCTION}

Coronal mass ejections (CMEs) are large-scale eruptive phenomena of the Sun and release a great deal of plasma and magnetic flux into the interplanetary space, consequently

\footnotetext{
${ }^{1}$ Key Laboratory of Solar Activity, National Astronomical Observatories, Chinese Academy of Sciences, Beijing 100012, China; shuhongyang@nao.cas.cn, zjun@nao.cas.cn

${ }^{2}$ Fuxian Solar Observatory, Yunnan Astronomical Observatory, Chinese Academy of Sciences, Kunming 650011, China; lz@ynao.ac.cn
} 
disturbing the space environment around the Earth (Gosling 1993; Webb et al. 1994). As identified in the white light observations, the structure of a CME consists of three parts, i.e., a bright leading front, a dark cavity, and a bright core (Illing \& Hundhausen 1983; Chen 2011). The dark cavity is generally deemed to be a twisted magnetic flux rope (Gibson et al. 2006; Riley et al. 2008). The inner bright core is widely believed to be filament matter suspended in flux rope dips (Guo et al. 2010; Jing et al. 2010). Filament structures are quite conspicuous in $\mathrm{H} \alpha$ observations (Hirayama 1985; Martin 1998; Lin et al. 2005) and their dynamic interactions can be caused by magnetic reconnection between the filamentcarrying magnetic fields (Török et al. 2011; Jiang et al. 2013). Detailed analyses reveal that magnetic flux ropes play a critical role in the formation and acceleration of CMEs (Patsourakos \& Vourlidas 2012; Cheng et al. 2013).

Magnetic flux ropes can emerge directly from below the photosphere into the upper atmosphere. Using continuous vector magnetograms from the Hinode satellite, Okamoto et al. (2008) found that two abutting regions with opposite polarities connected by strong horizontal magnetic fields first grew and then narrowed, and the orientations of the horizontal fields along the polarity inversion line changed from the normal polarity configuration to the inverse polarity one gradually. Moreover, there were significant blueshifts at the strong horizontal magnetic field area. They suggested that they observed a magnetic flux rope that was emerging from the sub-photosphere. Helical flux ropes can also be formed through magnetic reconnection between two bundles of J-shaped loops which have been frequently observed as sigmoidal structures in the extreme ultraviolet (EUV) and X-ray lines (e.g., Canfield et al. 1999; McKenzie \& Canfield 2008; Liu et al. 2010; Green et al. 2011). In simulations, magnetic reconnection between sheared loops are performed due to the imposed boundary movements, and thus can form magnetic flux ropes (Amari et al. 2000, 2003, 2011; Fan \& Gibson 2003, 2004; Aulanier et al. 2010). Moreover, using nonlinear force-free field models, magnetic flux ropes can be reconstructed from vector magnetic field observations (Canou et al. 2009; Canou \& Amari 2010; Guo et al. 2010, 2013; Jing et al. 2010; Su et al. 2011; Jiang et al. 2013; Inoue et al. 2013). After the launch of the Solar Dynamics Observatory (SDO; Pesnell et al. 2012), with the help of high-quality multi-wavelength data of the Atmospheric Imaging Assembly (AIA; Lemen et al. 2012), many authors have reported the existence of flux ropes in the observations (Cheng et al. 2012; Zhang et al. 2012a; Li \& Zhang 2013a, 2013b, 2013c; Patsourakos et al. 2013).

According to some previous observational studies, flux ropes are hot channels in the inner corona before and during solar eruptions (e.g., Zhang et al. 2012a; Cheng et al. 2012). They can be observed in high temperature lines (e.g., $131 \AA$ ), while invisible in low temperature lines (e.g., $171 \AA$ ). Using differential emission measure (DEM) analysis, Cheng et al. (2012) found that the temperature of twisted and writhed flux rope is higher than 8 
MK. However, in the studies of Li \& Zhang (2013a, 2013b) and Patsourakos et al. (2013), the flux ropes can be observed in all the seven EUV lines formed from 0.05 MK to $11 \mathrm{MK}$. Especially, for the two events investigated by Li \& Zhang (2013b), the flux ropes were tracked by erupting material, leading to the visibility of them, while they could not be detected in all wavelengths at the pre-eruption stage.

The New Vacuum Solar Telescope (NVST; Liu \& Xu 2011) is the most important facility of the Fuxian Solar Observatory in China. The diameter of NVST is $1 \mathrm{~m}$ and the pure aperture is 980 millimeter. One main goal of NVST is to image the Sun at high resolution. As one of the three channels ( $\mathrm{H} \alpha, \mathrm{TiO}$, and $\mathrm{G}$ band) being used now, $\mathrm{H} \alpha$ is used to observe magnetic structures in the chromosphere. In this Letter, we investigate in detail a flux rope tracked by a filament activation using NVST H $\alpha$ observations for the first time. Combined with the Helioseismic and Magnetic Imager (HMI; Scherrer et al. 2012) and AIA data, we also study the activation and the movement of the filament, and present the corresponding appearance of higher layers revealed in different EUV passbands.

\section{OBSERVATIONS AND DATA ANALYSIS}

The data used in this study were obtained by the NVST in $\mathrm{H} \alpha$ 6162.8 $\AA$ from 02:20:02 UT to 03:20:22 UT on 2013 February 1 . The $\mathrm{H} \alpha$ images are centered at $\mathrm{N}^{\circ} 1^{\circ} \mathrm{E} 37^{\circ}$ with a field-of-view (FOV) of $159^{\prime \prime} \times 159^{\prime \prime}$. The cadence of $\mathrm{H} \alpha$ observations is $12 \mathrm{~s}$ and the pixel size is $0^{\prime \prime} .162$. In addition, we adopt the $S D O /$ AIA multi-wavelength images observed from 02:00 UT to 03:30 UT and SDO/HMI line-of-sight (LOS) magnetograms from 00:00 UT to 03:30 UT. Among the 10 wavelengths of AIA, we choose $304 \AA, 171 \AA$, and $131 \AA$ data with a pixel size of $0^{\prime \prime} .6$ and a cadence of $12 \mathrm{~s}$. The HMI magnetograms have a spatial sampling of $0^{\prime \prime} .5$ pixel $^{-1}$ and a cadence of $45 \mathrm{~s}$. In addition, an NVST TiO image and an

HMI intensitygram observed at 03:10 UT are adopted for the coalignment of observations from different instruments.

The $\mathrm{H} \alpha$ data are dark current subtracted and flat field corrected to Level 1, and then reconstructed to Level $1+$ with the speckle masking method of Weigelt (1977). All the H $\alpha$ images are coaligned with each other by applying the image cross-correlation method. The AIA images and HMI magnetograms are aligned using the standard routine aia_prep.pro, and then derotated differentially to a reference time (02:20 UT, 2013 February 1). The HMI intensitygram and NVST $\mathrm{H} \alpha$ image at 03:10 UT are coaligned with the TiO image with the cross-correlation of obvious features. Then all the AIA and HMI data are coaligned with $\mathrm{H} \alpha$ images. 


\section{RESULTS}

Figure 1 shows the overview of the whole FOV H $\alpha$ image (left panel) and the simultaneous HMI magnetogram (right panel). The active region (AR) covered by the $\mathrm{H} \alpha$ observations is $\mathrm{AR} 11665$ at $\mathrm{N} 11^{\circ} \mathrm{E} 37^{\circ}$. The filament was located at the southeast of the main sunspot. The activation of the filament began at the northeast endpoint (outlined by square "1") and then the filament material moved toward the southwest direction, tracking out the preexisting flux rope.

\subsection{Activation of the filament}

At the area where the northeast end of the filament was located, the magnetic fields are shown in the top panels of Figure 2. The magnetic patches of positive and negative polarities moved toward each other (panel (a1)) and canceled gradually (panel (a2)). Using the inductive local correlation tracking method (ILCT; Welsch et al. 2004), we calculate the horizontal velocities of the photospheric magnetic fields. In panel (a2), the horizontal velocities of the negative and positive polarities represented by the red and blue arrows indicate that the negative fields were encountering the positive ones, resulting in the cancellation between them. At 02:28:55 UT, the magnetic fields, especially the negative polarity, had significantly disappeared due to the cancellation (see panel (a3)). The temporal evolution of the negative magnetic flux in the cancellation area is shown with the read curve in panel (a3). We can see that, from 00:13:55 UT to 02:28:55, the unsigned negative flux persistently decreased by $26 \%$ from $3.5 \times 10^{19} \mathrm{Mx}$ to $2.6 \times 10^{19} \mathrm{Mx}$. During the cancellation process, the filament was activated, exhibiting as rising and expanding (panels (b1) - (b3)). At 02:29:19 UT, the fine structures of the filament can be obviously identified and delineated with dashed curves in panel (b3). The crossed threads indicate that the filament was twisted. The bottom panels are the simultaneous AIA $304 \AA$ images corresponding to the $\mathrm{H} \alpha$ observations. At 02:20:19 UT, there was a dark feature (denoted by the black arrow in panel $(\mathrm{c} 1)$ ) at the filament location. While only about 7 min later, a jet-like brightening appeared (denoted by the black arrow in panel (c2)). Then the brightening feature expanded significantly in size, as shown in panel (c3). The jet-like brightening is commonly considered as a signature of magnetic reconnection. 


\subsection{Filament material flow tracking out the twisted flux rope}

After activation, filament material flowed to the southwest direction and tracked out pre-existing helical threads (see the left column of Figure 3 and Movie 1). At 02:35:22 UT, the filament material was mainly located near the northeast end (denoted by the arrow in panel (a)). About 7 min later, the material moved to a farther position (denoted by the arrow in panel (b)). At that moment, the twisted structure outlined by the dark material was more pronounced compared with that $7 \mathrm{~min}$ ago. This part of flux rope is outlined by the blue and red curves in panel (b). The material went on flowing and reached the place denoted by the arrow (see panel (c)) at 02:55:57 UT. Another section of the flux rope was consequently tracked out by the filament material, and outlined by the blue and red curves. In order to show the entire flux rope whose different sections were filled with dark material at different moments, a composite $\mathrm{H} \alpha$ image is presented in panel (d). In panel (d), the sub-regions outlined by two green quadrangles were observed at 02:39:12 UT and 02:42:50 UT, respectively. The rest background image was observed at 02:55:57 UT. The entire flux rope exhibits as a twisted structure figured out by the dark filament material and connects the positive fields at the northeast and the negative fields at the southwest (the red and the blue contours in panel $(\mathrm{d}))$. The approximate length of the twisted flux rope is 75 $\mathrm{Mm}$. Combined with the observations at different times, the twist configuration of the flux rope tracked out by the material flow along the helical threads is roughly sketched out and overlaid on the magnetogram obtained at 02:55:55 UT (see the blue and red thick curves in panel (e)). The left part of the flux rope was tracked out at 02:42:50 UT, and the right part was tracked out at 02:55:57 UT. Moreover, the right section of the flux rope can be well identified in AIA $304 \AA$ line (see the blue and green curves in Figure 4 (b2)). We estimate that the twist of the flux rope is about $1 \pi$. To show the material flow clearly, a space-time plot along curve "A-B" marked in panel (b) is shown in panel (f). The plot reveals that the filament material moved from "A" to "B" and the mean velocity is $31.1 \mathrm{~km} \mathrm{~s}^{-1}$.

\subsection{Appearance in multi-wavelength images}

We also examine the multi-wavelength images obtained by AIA and display $304 \AA, 171$ $\AA$, and $131 \AA$ images before and during the filament activation in Figure 4 (see also Movies 2, 3, and 4). At 02:20 UT, before the activation, the filament appeared as a thin dark structure in the $\mathrm{H} \alpha$ image (panel (a1)). In $304 \AA$ image (panel (b1)), there was a faint dark channel corresponding to the $\mathrm{H} \alpha$ filament, while there was no distinct dark or bright structure in

$171 \AA$ (panel (c1)) and $131 \AA$ (panel (d1)) images. At 02:46 UT, during the activation, the $\mathrm{H} \alpha$ filament expanded and contained many dark threads. In $304 \AA$, the flux rope displays 
as a partial bright and partial dark structure. While in $171 \AA$ and $131 \AA$ images, there were bright structures at the $\mathrm{H} \alpha$ filament location.

The cuts of multi-wavelengths along slice "A-B" marked in Figure 4 are presented in the higher panels of Figure 5. The gray shadows mark the filament width identified in $\mathrm{H} \alpha$ images. Panel (a) shows the cuts at 02:20 UT. In $304 \AA$ cut, the section corresponding to filament, i.e., the gray section in $\mathrm{H} \alpha$ cut, only shows a slight lower emission. While in $171 \AA$ and $131 \AA$ cuts, there is no distinct high or low emission at the corresponding section. Panel (b) shows the cuts at 02:46 UT. The $\mathrm{H} \alpha$ cut shows that there are many dark structures (see the gray section). The cuts of $304 \AA, 171 \AA$, and $131 \AA$ show much higher emissions. Panel (b) shows a striking anti-correlation between $\mathrm{H} \alpha$ and EUV lines, i.e., peaks of the former correspond to maxima of the latter and vice versa. As revealed by the $\mathrm{H} \alpha$ cut (black curve in panel (b)), four threads of the flux rope are well-resolved. In order to measure their width, we fit the sections of the four threads using Gaussian fitting. The widths of them are 1.19 $\mathrm{Mm}, 0.62 \mathrm{Mm}, 1.63 \mathrm{Mm}$, and 1.00 Mm, respectively. Their average width of its individual threads is about $1.11 \mathrm{Mm}$.

As shown in panels (c1) and (c2) of Figure 4, there are many bright coronal loops overlying the filament location. To examine the influence of the filament activation on the coronal loops, a space-time plot along curve "C-D" (marked in Figure 4) is displayed in panel (c) of Figure 5. We can see that, from 02:00 UT to 03:30 UT, the coronal loops (bright structures) were quite steady.

\section{CONCLUSIONS AND DISCUSSION}

Based on the NVST H $\alpha$ observations for the first time, we study in detail a flux rope which was tracked by a filament activation. The filament material was initially located at one end of the flux rope, and the filament was activated due to the magnetic field cancellation. The activated filament then rose and flowed along the flux rope, tracking out the twisted structure. The length of the flux rope is about $75 \mathrm{Mm}$, the average width of its individual threads is $1.11 \mathrm{Mm}$, and the estimated twist is $1 \pi$. The tracked flux rope appeared as a dark structure in $\mathrm{H} \alpha$ images, a partial dark and partial bright structure in $304 \AA$, while as bright structures in $171 \AA$ and $131 \AA$ images. During this process, the overlying coronal loops were quite steady since the filament was confined within the flux rope and did not erupt successfully.

The bright core of a CME is thought to be cool filament matter which is suspended in the dips of magnetic flux (e.g., Xia et al. 2012; Zhang et al. 2012b). As revealed by 
the event in the present study, the filament is closely related with the flux rope. It seems that, the steady filament is only located at one section of the flux rope. However, instead of being suspended in the dips of twisted magnetic flux, the filament material is confined within the helical threads. Only when the filament is activated, the filament material then can flow easily along the threads and thus track out the twisted structure of the flux rope. We tend to support a picture of a pre-existing flux rope that was tracked out by filament material activated by magnetic flux cancellation. However, since the observation duration of the present event is very short and the field-of-view is not large enough, we cannot exclude the formation of new flux rope due to flux cancellation, which is a popular mechanism for flux rope formation (e.g., van Ballegooijen \& Martens 1989).

As studied by Zhang et al. (2012a) and Cheng et al. (2012), flux ropes could be clearly observed before and during eruptions and were only detected in hot temperature passbands. While in this study, the flux rope was only observed during the filament activation instead of before the activation, which is similar to the study of Li \& Zhang (2013b). The flux rope was tracked out by the filament material and detected in low temperature (e.g., $304 \AA$ ) and high temperature (e.g., $131 \AA$ ) lines, consistent with the results of Li \& Zhang (2013a, 2013b). Moreover, our results show that there exists a striking anti-correlation between $\mathrm{H} \alpha$ and EUV lines (see Figure 5(b)). This could imply some mild heating of cool filament material into coronal temperatures during the filament activation (e.g., Landi et al. 2010). The heating should not be flare-like, reaching temperatures of $10 \mathrm{MK}$ or more. This can be demonstrated by the almost identical cuts in the AIA $171 \AA$ and $131 \AA$ channels (the $131 \AA$ channel bandpass besides containing a flare peak at around $10 \mathrm{MK}$, also contains a "warm" peak at several 0.1 MK, similar to the main peak of the $171 \AA$ channel).

In some former studies (Li \& Zhang 2013a, 2013b) and also this study, flux ropes were observed in different EUV lines and appeared as bright structures (see Figures 4(c2), and 4(d2)) or partial bright structures (see Figure 4(b2)), indicating the co-existence of hot and cool components in flux ropes. In contrary, the twisted flux rope in $\mathrm{H} \alpha$ images consists of dark threads, which is different from the appearance in EUV images. For the flux ropes studied by Li \& Zhang (2013b), the approximate length is $570 \mathrm{Mm}$. They measured the width of individual thread of flux ropes and found that the average width is $1.16 \mathrm{Mm}$. However, the length of flux rope in the present study is only $75 \mathrm{Mm}$, much smaller than those studied by Li \& Zhang (2013b). In the $\mathrm{H} \alpha$ images, the fine-scale threads can be generally resolved, and their mean width is determined to be $1.11 \mathrm{Mm}$, consistent with the result of Li \& Zhang (2013b).

This work is supported by the Outstanding Young Scientist Project 11025315, the National Basic Research Program of China under grant 2011CB811403, the CAS Project 
KJCX2-EW-T07, the Strategic Priority Research Program-The Emergence of Cosmological Structures of the Chinese Academy of Sciences (Grant No. XDB09000000), and the National

Natural Science Foundations of China (11203037, 11221063, 11373004, and 11303049). The data are used courtesy of NVST and SDO science teams.

\section{REFERENCES}

Amari, T., Aly, J.-J., Luciani, J.-F., Mikic, Z., \& Linker, J. 2011, ApJ, 742, L27

Amari, T., Luciani, J. F., Aly, J. J., Mikic, Z., \& Linker, J. 2003, ApJ, 585, 1073

Amari, T., Luciani, J. F., Mikic, Z., \& Linker, J. 2000, ApJ, 529, L49

Aulanier, G., Török, T., Démoulin, P., \& DeLuca, E. E. 2010, ApJ, 708, 314

Canfield, R. C., Hudson, H. S., \& McKenzie, D. E. 1999, Geophys. Res. Lett., 26, 627

Canou, A., \& Amari, T. 2010, ApJ, 715, 1566

Canou, A., Amari, T., Bommier, V., et al. 2009, ApJ, 693, L27

Chen, P. F. 2011, Living Reviews in Solar Physics, 8, 1

Cheng, X., Zhang, J., Ding, M. D., et al. 2013, ApJ, 769, L25

Cheng, X., Zhang, J., Saar, S. H., \& Ding, M. D. 2012, ApJ, 761, 62

Fan, Y., \& Gibson, S. E. 2003, ApJ, 589, L105

Fan, Y., \& Gibson, S. E. 2004, ApJ, 609, 1123

Gibson, S. E., Foster, D., Burkepile, J., de Toma, G., \& Stanger, A. 2006, ApJ, 641, 590

Gosling, J. T. 1993, J. Geophys. Res., 98, 18937

Green, L. M., Kliem, B., \& Wallace, A. J. 2011, A\&A, 526, A2

Guo, Y., Ding, M. D., Cheng, X., Zhao, J., \& Pariat, E. 2013, ApJ, 779, 157

Guo, Y., Schmieder, B., Démoulin, P., et al. 2010, ApJ, 714, 343

Hirayama, T. 1985, Sol. Phys., 100, 415

Illing, R. M. E., \& Hundhausen, A. J. 1983, J. Geophys. Res., 88, 10210 
Inoue, S., Hayashi, K., Shiota, D., Magara, T., \& Choe, G. S. 2013, ApJ, 770, 79

Jiang, C., Feng, X., Wu, S. T., \& Hu, Q. 2013, ApJ, 771, L30

Jiang, Y., Hong, J., Yang, J., et al. 2013, ApJ, 764, 68

Jing, J., Yuan, Y., Wiegelmann, T., et al. 2010, ApJ, 719, L56

Landi, E., Raymond, J. C., Miralles, M. P., \& Hara, H. 2010, ApJ, 711, 75

Lemen, J. R., Title, A. M., Akin, D. J., et al. 2012, Sol. Phys., 275, 17

Li, L. P., \& Zhang, J. 2013a, A\&A, 552, L11

Li, T., \& Zhang, J. 2013b, ApJ, 770, L25

Li, T., \& Zhang, J. 2013c, ApJ, 778, L29

Lin, Y., Engvold, O., Rouppe van der Voort, L., Wiik, J. E., \& Berger, T. E. 2005, Sol. Phys., 226,239

Liu, R., Liu, C., Wang, S., Deng, N., \& Wang, H. 2010, ApJ, 725, L84

Liu, Z., \& Xu, J. 2011, First Asia-Pacific Solar Physics Meeting, ASI Conference Series, Edited by Arnab Rai Choudhuri \& Dipankar Banerjee, 2, 9

Martin, S. F. 1998, Sol. Phys., 182, 107

McKenzie, D. E., \& Canfield, R. C. 2008, A\&A, 481, L65

Okamoto, T. J., Tsuneta, S., Lites, B. W., et al. 2008, ApJ, 673, L215

Patsourakos, S., \& Vourlidas, A. 2012, Sol. Phys., 281, 187

Patsourakos, S., Vourlidas, A., \& Stenborg, G. 2013, ApJ, 764, 125

Pesnell, W. D., Thompson, B. J., \& Chamberlin, P. C. 2012, Sol. Phys., 275, 3

Riley, P., Lionello, R., Mikić, Z., \& Linker, J. 2008, ApJ, 672, 1221

Scherrer, P. H., Schou, J., Bush, R. I., et al. 2012, Sol. Phys., 275, 207

Su, Y., Surges, V., van Ballegooijen, A., DeLuca, E., \& Golub, L. 2011, ApJ, 734, 53

Török, T., Chandra, R., Pariat, E., et al. 2011, ApJ, 728, 65

van Ballegooijen, A. A., \& Martens, P. C. H. 1989, ApJ, 343, 971 
Webb, D. F., Forbes, T. G., Aurass, H., et al. 1994, Sol. Phys., 153, 73

Weigelt, G. P. 1977, Optics Communications, 21, 55

Welsch, B. T., Fisher, G. H., Abbett, W. P., \& Regnier, S. 2004, ApJ, 610, 1148

Xia, C., Chen, P. F., \& Keppens, R. 2012, ApJ, 748, L26

Zhang, J., Cheng, X., \& Ding, M.-D. 2012a, NatCo, 3, 747

Zhang, Q. M., Chen, P. F., Xia, C., \& Keppens, R. 2012b, A\&A, 542, A52 

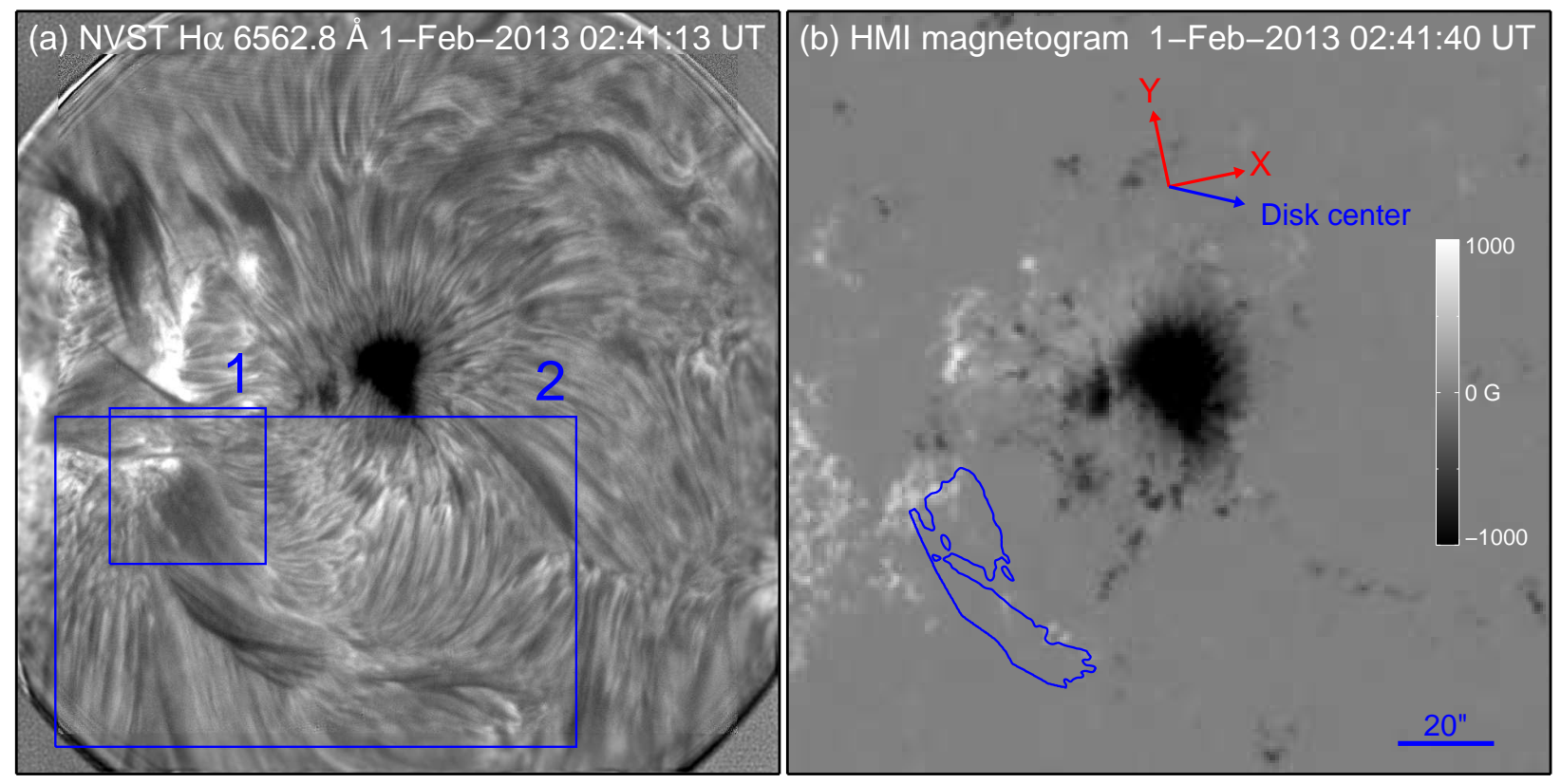

Fig. 1.- NVST H $\alpha$ image (left panel) and HMI line-of-sight magnetogram (right panel). Square "1" outlines the FOV of Figures 2 (b1)-(c3), and rectangle "2" delineates the FOV of Figures 3 and 4 . The blue curve in panel (b) is the contour of the filament during activation. 


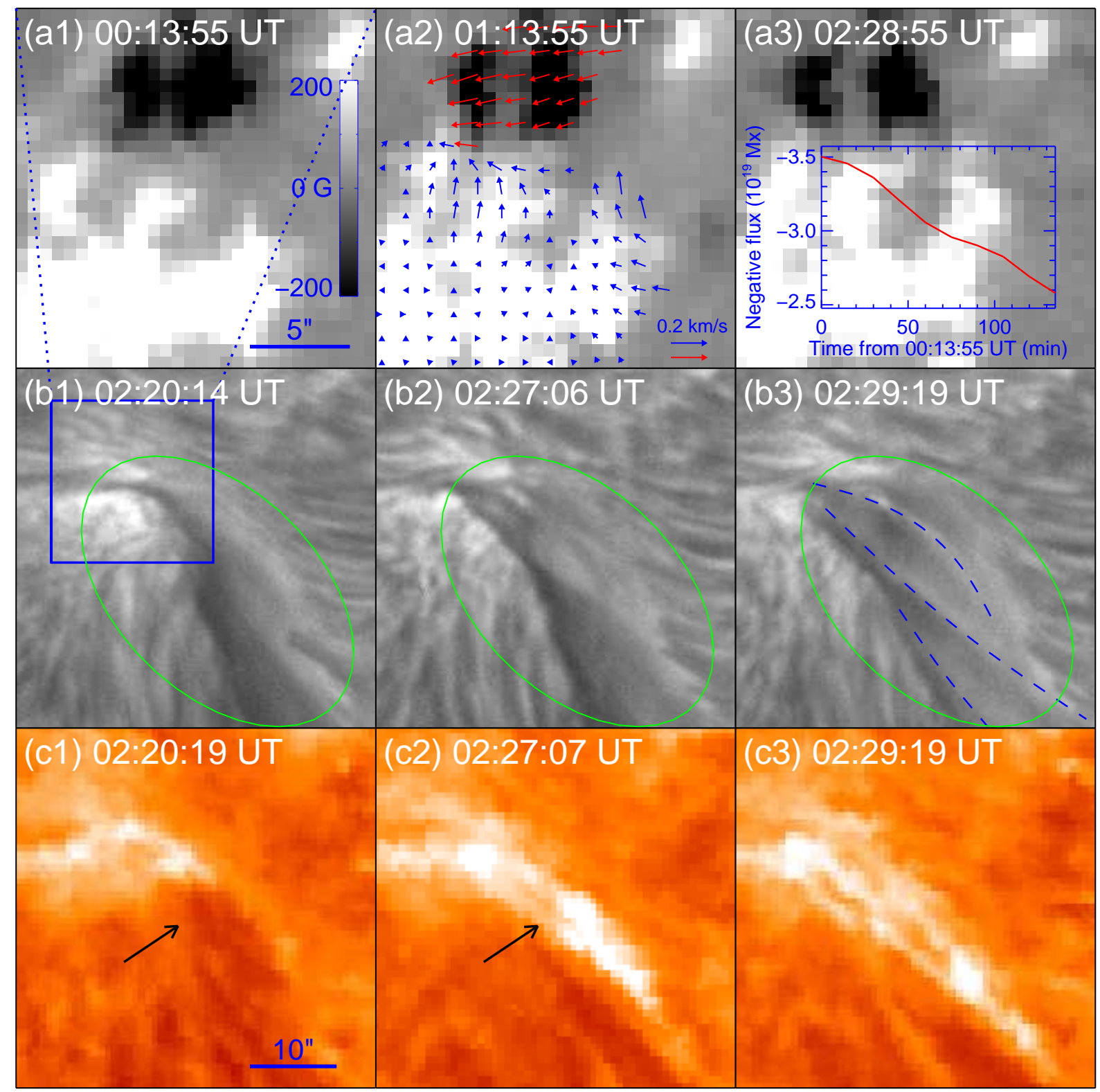

Fig. 2.- Top panels: magnetograms showing the cancellation of opposite polarities. Middle panels: $\mathrm{H} \alpha$ images displaying the activation of the filament. Bottom panels: AIA images exhibiting brightening in $304 \AA$. The red and blue arrows in panel (a2) represent the horizontal velocities of negative and positive magnetic fields, respectively. The red curve in panel (a3) displays the temporal evolution of the negative magnetic flux in the area where cancellation takes place. The square in panel (b1) outlines the FOV of panels (a1-a3). The ellipses in panels (b1-b3) outline the filament location, and the arrows in panels (c1-c2) denote the EUV brightening. The dashed curves in panel (b3) delineate three threads of the activated filament. 


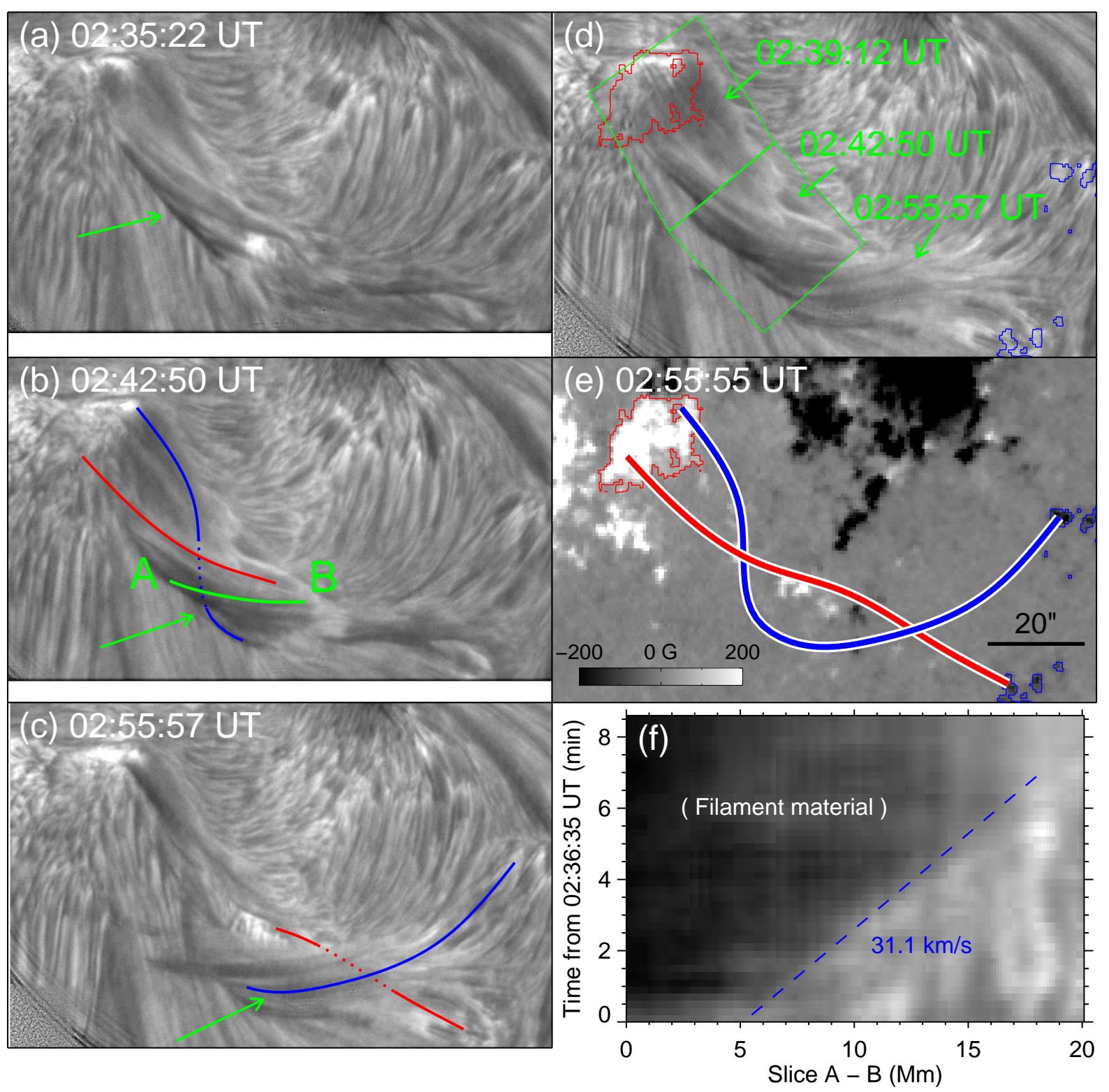

Fig. 3.- Panels (a-c): H $\alpha$ images showing the process of the filament activation. Panel (d) is a composite $\mathrm{H} \alpha$ image showing the whole flux rope tracked by the filament material, and panel (e) is the corresponding photospheric magnetogram. Panel (f) is the space-time plot showing the dark filament material flow along curve "A-B" marked in panel (b). The arrows in panels $(\mathrm{a}-\mathrm{c})$ denote the filament locations at different stage. The red and blue thin curves in panels (b) and (c) outline the left part and right part of the flux rope, respectively, while the thick curves in panel (e) display the whole twist configuration of the flux rope. The red and blue thin curves in panels (d) and (e) indicate the flux rope footpoints with positive and negative polarities, respectively. 


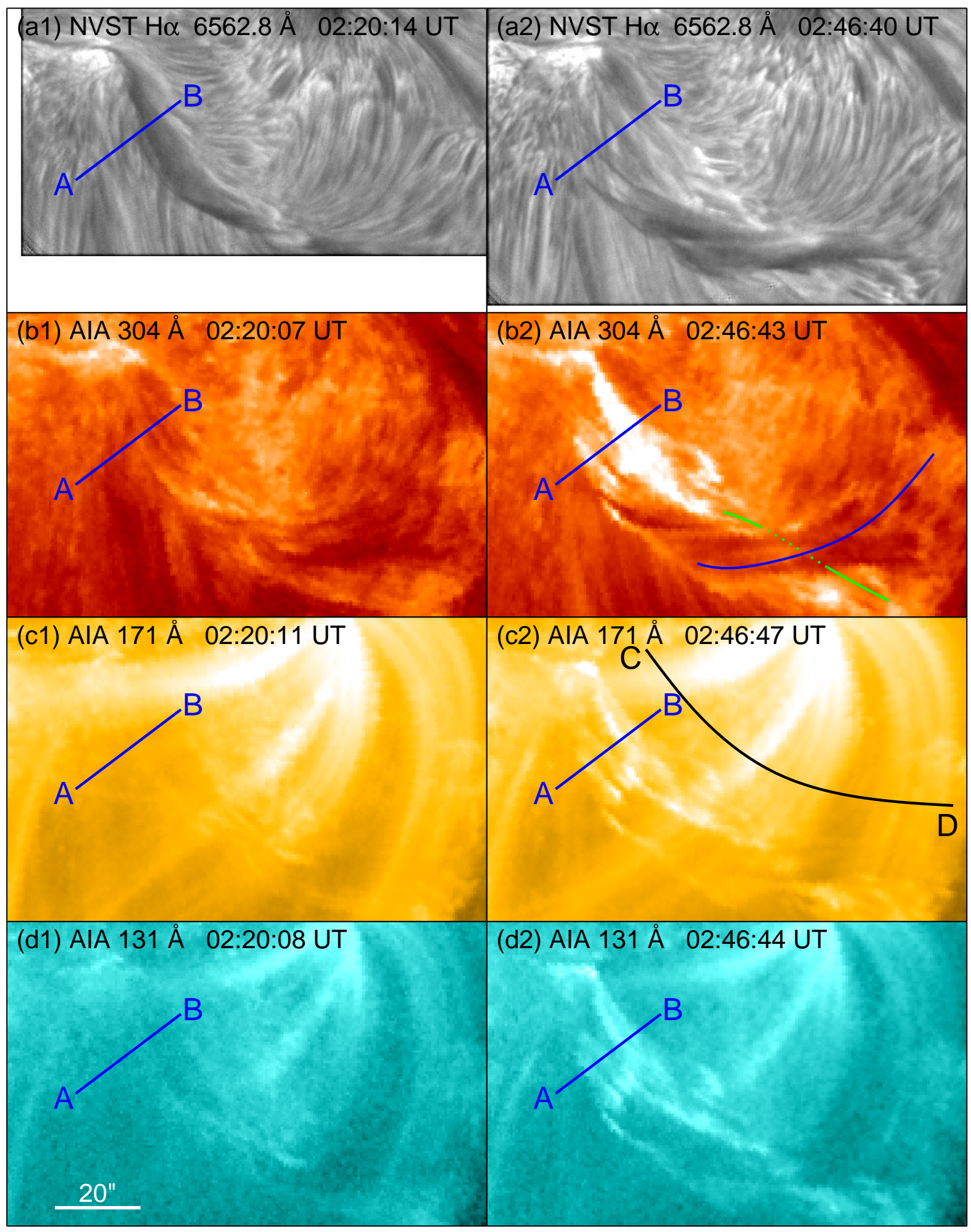

Fig. 4.- Multi-wavelength images before (left panels) and during (right panels) the filament activation. Lines "A"-"B" and curve "C"-"D" mark the positions along which the cuts and the space-time plot in Figure 5 are obtained. The green and blue curves in panel (b2) outline the right part of the flux rope that can be well identified in AIA $304 \AA$ line. 

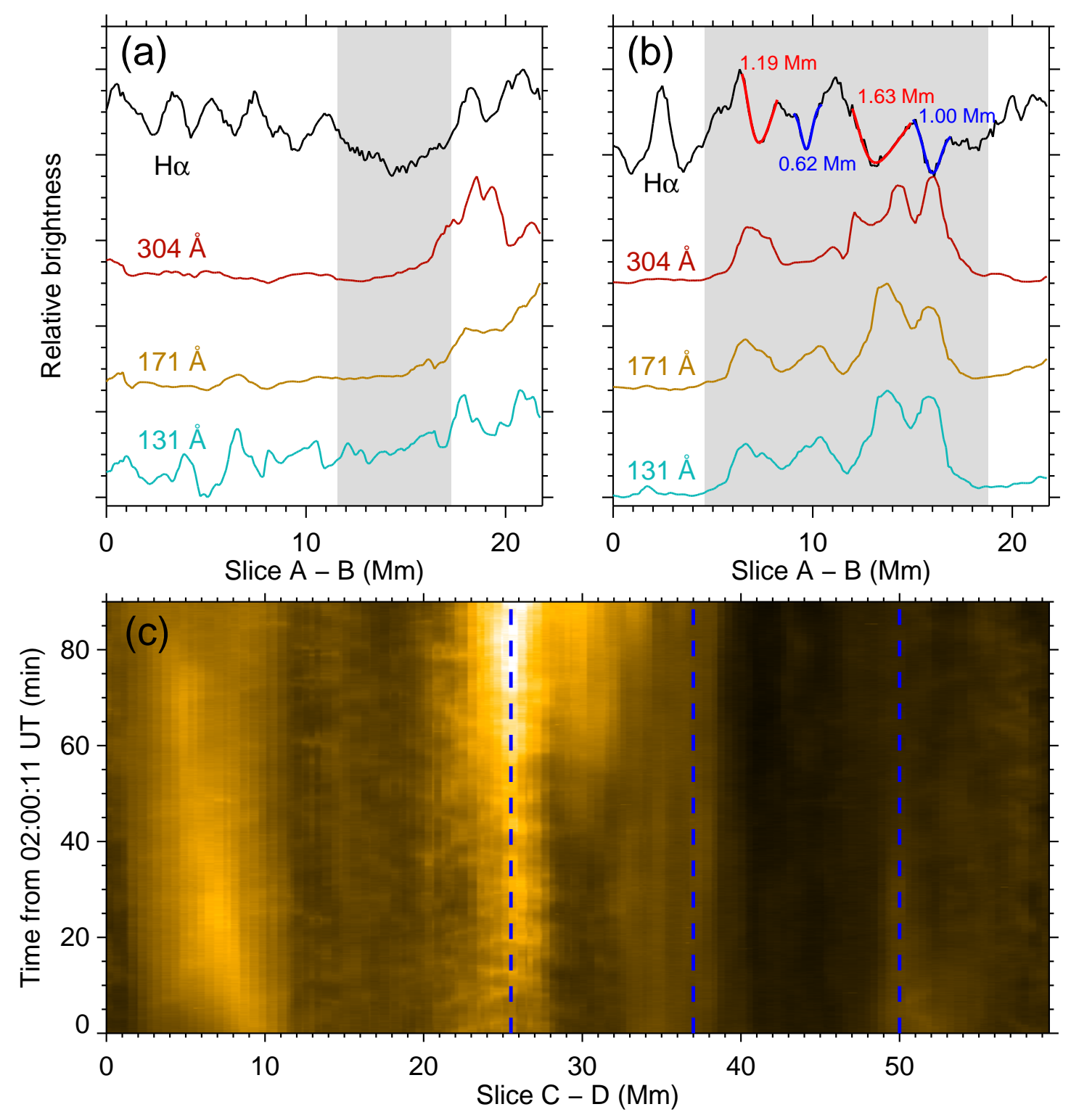

Fig. 5.- Panels (a) and (b): relative brightness of multi-wavelengths along slice "A-B" (see Figure 4) at 02:20 UT and at 02:46 UT, respectively. Panel (c): space-time plot along curve "C-D" marked in Figure 4. The red and blue curves overlaid on the black curve in panel (b) are Gaussian fittings of four flux rope threads. 